\title{
Stefan Menzel, Hōgaku. Traditionelle japani- sche Musik im 20. Jahrhundert, Hildesheim: Olms 2015
}

Schlagworte/Keywords: 20. Jahrhundert; 20th century; cross-cultural influence; ethnomusicology; Ethnomusikologie; hōgaku; interkulturelle Rezeption; Japan; traditional music; traditionelle Musik

Hōgaku - die traditionelle Musik Japans ${ }^{1}$ - ist keineswegs nur eine Museumskultur, sie büßte durch die seit der Meiji-Restauration (ab 1868) immer stärkere Auseinandersetzung mit der westlichen Kultur und folglich auch westlichen Musik keineswegs ihre kreative Lebendigkeit ein. Doch nur selten wurde die Entwicklung von hōgaku nach 1868 von westlichen Autoren thematisiert. Eine relativ ausführliche Darstellung findet sich in den letzten beiden Kapiteln von Eta Harich-Schneiders grundlegendem Werk über japanische Musik, ${ }^{2}$ doch liegt das Erscheinen dieses Buches nicht nur mittlerweile fast ein halbes Jahrhundert zurück, die Autorin, die aus ihrer Präferenz für die älteren Genres japanischer Musik - wie gagaku und $n \bar{o}-$ und ihrer Geringschätzung für die meiste Musik der Tokugawazeit (1600/03-1868) auch sonst keinen Hehl machte, widmete sich vorwiegend der Transformation, der gagaku in der MeijiZeit unterlag, und deutet die Entwicklung in anderen Genres nur an. ${ }^{3}$ Hingegen mangelt es an Literatur zur älteren hōgaku nicht. ${ }^{4}$

$1 \quad$ Hōgaku ist, wie viele auf japanische traditionelle Musik bezugnehmende Begriffe, leidvoll ungenau definiert und umfasst eine Vielzahl höchst unterschiedlicher Genres. Menzel beschreibt die Problematik des Begriffs eingehend (6f.) - so besteht z. B. nicht nur theoretisch die Möglichkeit, dass selbst bestimmte Formen des J-Pop als hōgaku bezeichnet werden können.

2 Harich-Schneider 1973, 533-597. In japanischer Sprache wird die Entwicklung von hōgaku seit der Meiji-Zeit ausführlich von Kikkawa Eishi in den letzten Kapiteln seiner Geschichte der japanischen Musik behandelt (Kikkawa 1965, 354ff.).

3 Naturgemäß behandelt auch z. B. Ingrid Fritsch (2005) in ihrer Arbeit die Entwicklung von hōgaku im 20. Jahrhundert insofern, als die
So füllt die außerordentlich fakten- und materialreiche Arbeit über die Geschichte von hōgaku seit 1868, die Stefan Menzel vorlegt, eine empfindliche Lücke. Besonderes Gewicht legt Menzel auf die Schilderung soziokultureller Aspekte und die diskursive Einbettung musikalischer Entwicklungen, der im westlichen Schrifttum über japanische Musik sonst meist wenig Beachtung geschenkt wird. Gerade darin verdient Menzels Studie über seinen Gegenstand hinausweisendes Interesse. Doch fehlen auch musikalische Analysen besonders aussagekräftiger Beispiele nicht. Sicher ließen sich an diese Analysen weiterführende Überlegungen anknüpfen, die z. B. das Phänomen der Begegnung unterschiedlicher musikalischer Denkweisen aus musiktheoretischer Sicht beleuchten.

Das erste Kapitel (»Der Weg in die ästhetische Krise«) zeichnet differenziert die politischen und gesellschaftlichen Verwerfungen der frühen Meiji-Zeit nach, die sich zwangsläufig in der Auseinandersetzung mit der gleichermaBen als Bedrohung wie als Chance wahrgenommenen westlichen Kultur ergaben und die umfassende Wirkungen auch auf Musikausübung und -verständnis hatten - viele traditionelle Genres gerieten am Anfang der Meiji-Zeit durch Verbot sie bisher schützender Institutionen (wie der Blindengilde tōdō-za) bzw. durch Wegfallen staatlicher Förderung in eine schwere Krise. Langfristigeren Einfluss übte die Einführung des shōka, des an westlichen Modellen

Tōzan-Schule der shakuhachi erst 1896 gegründet wurde. Doch hier handelt es sich lediglich um die Darstellung eines Genres.

4 Stellvertretend sei für einen umfassenden Überblick Provine/Tokumaru/Witzleben (2002, 567-834) genannt. 
ausgerichteten Singunterrichts ${ }^{5}$ in der Schule aus: »Ein jeder Japaner, der seit den 1880er Jahren eingeschult worden war, durchlief $[\ldots]$ ästhetische Umerziehung،, wurde an westliche Melodik und Harmonik gewöhnt" (47). Diese und ähnliche erziehungspolitische Maßnahmen wurden von den Verantwortlichen durch den Verweis auf angebliche Defizite der traditionellen japanischen Musik gerechtfertigt. Dass sich darin nicht nur eine Reaktion auf die als überlegen wahrgenommene westliche Kultur, sondern auch konfuzianisch geprägtes Denken niederschlug, gehört zu den wesentlichen Beobachtungen, die Menzel hier vermittelt. So findet sich in den Kulturdiskussionen der MeijiZeit immer wieder der Topos von den sschädlichen gesellschaftlich-moralischen Wirkungen der zokkyoku, der traditionellen , Unterhaltungsmusikı, zu der man auch die Musik populärer Theaterformen wie kabuki und bunraku zählte.

Im zweiten Kapitel (»Die Bewältigung der Moderne«) wird die Geschichte von hōgaku bis 1945 vor allem anhand der Begegnungen zwischen traditioneller und westlicher Musik dargestellt. Naturgemäß kommt dem Schaffen des Koto- und Shamisen-Virtuosen Miyagi Michio ${ }^{6}$ (1894-1956) große Bedeutung zu. Menzel leuchtet den Kontext dieser zentralen Figur aus: die Meiji shinkyoku, die westlich orientierten sneuen Stücke der Meiji-Zeit s des KotoRepertoires, von denen heute nur noch ein kleiner Bruchteil erhalten ist und an die Miyagi in seinen ersten Werken anknüpft; die Rolle von Miyagis Umfeld, seines 'Managers Y Yoshida Seifū, des Musikwissenschaftlers Tanabe Hisao und des Musikkritikers und späteren Rundfunkredakteurs Machida Kashō, die Miyagis Musik zur nötigen Medienpräsenz verhalfen - Miyagi Michio war sder richtige Mann zur richtigen Zeitı, der als Projektionsfigur für eine damals von vielen Intellektuellen verspürte Sehnsucht nach einem Brückenschlag zwi-

5 Shōka bezeichnet sowohl den Singunterricht als auch die speziell hierfür komponierten (Schul-)Lieder.

6 Japanische Namen werden in der in Japan üblichen Reihenfolge sNachname Vorname angegeben. schen westlicher und traditioneller japanischer Kultur diente. Für Musiktheoretiker*innen interessant ist Menzels Hinweis auf eine sdoppelte Codierung - double function form - in einigen von Miyagis Werken (100), die vielleicht einen analytischen Ansatz bereitstellt, in der scheinbaren Einfachheit dieser Werke einen komplexeren Hintergrund aufzuzeigen. Haru no umi, das wohl bekannteste Stück von Miyagi, lässt sich z. B. einerseits als dreiteilige Liedform verstehen, nimmt aber auch Bezug auf typische Formelemente japanischer sōkyokuMusik.

Fast noch spannender ist eine Fußnote (94, Fn. 82), die eine meines Wissens bisher nur in Ansätzen verfolgte Strategie zur theoretischen Durchdringung traditioneller japanischer Musik aufzeigt. Ausgehend davon, dass die meisten Genres fast theorielos unterrichtet werden, sieht Menzel als Grundbausteine der Musik nicht abstrakte Skalen oder Modi an, sondern typische Melodiewendungen, die beim Erlernen der Musik verinnerlicht werden. Freilich dürften bei konsequenter Umsetzung dieser Idee in analytische Techniken schwierige methodische Probleme auftreten. ${ }^{7}$

Des Weiteren beschreibt Menzel verschiedene Versuche der 1930er Jahre, hōgaku mit Orchestermusik in Verbindung zu bringen (der wohl bekannteste an solchen Versuchen beteiligte Komponist war Yamada Kōsaku). Der stark wechselnde Erfolg solcher Experimente ebenso wie die sich daran anschließenden zum Teil höchst leidenschaftlichen Diskussionen legen Zeugnis von der Verflochtenheit politischer,

7 Vor einigen Jahren fand eine Art ,Wiederentdeckung - oder Re-Evaluation - von Machida Kashōs musiktheoretischer Schrift Shamisenseikyoku ni okeru senritsukei no kenkyū [Studien zu Patterns in shamisen-begleiteter Gesangsmusik] statt (Machida 1956), vgl. Yamada/Ōkubo 2015. Die in diesem Buch vertretenen Aufsätze zeigen immerhin einige Folgerungen, die sich im Fahrwasser des von Menzel beschriebenen Ansatzes ergeben könnten, sie verdienen bei musiktheoretischer Auseinandersetzung mit traditioneller japanischer Musik Berücksichtigung. Daneben sei auch auf die Forschung von Yakō Masato verwiesen (z. B. Yakō 2002). 
sozialer und ästhetischer Diskurse ab, in die sich im Verlauf des Zweiten Weltkriegs zunehmend nationalistische Aspekte mischten.

Das dritte Kapitel (»Hōgaku nach 1945«) schließlich gilt der Entwicklung von hōgaku nach dem Zweiten Weltkrieg. Kurzporträts von drei Musiker*innen stellen grundsätzliche Positionen dar: Auf der einen Seite des Spektrums steht der >Traditionalist` Yamaguchi Gorō (shakuhachi), der sich fast ausschließlich der Pflege des klassischen Repertoires widmete; auf der andern Seite Yamamoto Hōzan (ebenfalls shakuhachi), der mit westlich orientierten Komponisten wie Hirose Ryōhei und Jazzmusikern zusammenarbeitete; und schließlich Miyagi Kiyoko (koto und shamisen), Nichte von Miyagi Michios zweiter Frau, die vor allem Miyagi Michios Erbe weiterführte und damit gewissermaßen eine Position der sgemäßigten Modernes innerhalb von hōgaku vertrat.

Die Entwicklung von hōgaku nach dem Zweiten Weltkrieg wurde wesentlich durch einige neue Institutionen geprägt. Eine dieser Institutionen war der Hōgaku-Fachbereich der Tokyo University of the Arts (Tōkyō Geijutsu Daigaku). Menzel schildert ausführlich die politischen Kontroversen, die sich daran entzündeten: Der 1936 erstmalig eingerichtete Fachbereich sollte laut einem Reformpapier der Hochschulleitung aus dem Jahre 1945 abgeschafft werden; der Musikwissenschaftler Kikkawa Eishi stand an der Spitze einer Protestbewegung, die vehement für seine Erhaltung eintrat. Am Ende traten sowohl der Rektor der Tōkyō Geijutsu Daigaku, der zu den Befürwortern einer Abschaffung des Fachbereiches gehörte, als auch Kikkawa Eishi von ihren Ämtern zurück, doch fand eine umfassende inhaltliche Reform des Fachbereiches statt. Hier macht Menzel deutlich, dass Kikkawas auch ins Deutsche übersetzte Schrift Vom Charakter der japanischen Musik ${ }^{8}$ zunächst vor allem in diesem Zusammenhang von zentraler Bedeutung war. Es handelt sich bei diesem Buch, wenn man so will, um eine kulturpolitische Kampfschrift, die nicht zuletzt der Wiedereinrichtung des Hōgaku-Fachbereiches an der wichtigsten japanischen Musikausbildungsstätte dienen sollte.
Der Einfluss dieses im Jahr 1949 neu eingerichteten Fachbereichs auf die Hōgaku-Szene erwies sich in der Folgezeit als überaus groß. Während bis dahin die soziale Struktur von hōgaku fast ausschließlich durch streng hierarchische Traditionslinien (ryūha) definiert worden war, entstand nun durch eine akademische Institution ein neuer Raum, in dem Begegnungen zwischen verschiedenen Genres, vor allem aber auch Begegnungen zwischen westlich ausgebildeten Komponist*innen und HogakuInterpret*innen möglich wurden.

Fast noch einflussreicher war das vom NHK (Nippon Hōsō Kyōkai, Japan Broadcasting Corporation) ins Leben gerufene Ausbildungsprogramm NHK hōgaku ginōsha ikusei kai (Technisches Schulungsprogramm für den HōgakuNachwuchs), das von 1955 bis 2010 bestand und insgesamt etwa 2000 Absolvent*innen hervorbrachte. Auch dieses Ausbildungsprogramm trug dazu bei, Alternativen zur RyūhaStruktur zu entwickeln und die Entstehung neuer Kompositionen anzuregen.

Was sich in den Ausbildungsprogrammen der Tokyo University of the Arts und des NHK anbahnte, führte zu einigen tiefgreifenden Veränderungen in der sozialen Struktur von hōgaku - z. B. zur Entstehung von traditionelle Ryūha-Grenzen überschreitenden Ensembles und Netzwerken (wie z. B. Hōgaku yonin no kai, ein Ensemble von vier HōgakuMusiker*innen, die insbesondere als Auftraggeber*innen neuer Kompositionen für HōgakuInstrumente dauerhaften Einfluss auf die Entwicklung von hōgaku hatten, und Pro Musica Nipponia, ein weit über 1000 HōgakuMusiker*innen umfassendes Künstlernetzwerk), zur Entstehung auch von Wettbewerben und Festivals, einer wachsenden Medienpräsenz (nicht zuletzt im Rahmen des `Hōgaku-Boom der 1960er und 1970er Jahre, dem wir viele zum Teil äußerst aufwendig gestaltete Schallplatteneditionen verdanken) etc. Solche Erscheinungen sind teilweise durchaus analog zum westlichen Musikbetrieb, mussten aber für hōgaku erst erobert werden. Wie Menzel zeigt, gingen die Impulse für neue Kompositionen im hōgaku vor allem von Interpret*innen und Ensembles aus; sie bildeten gewissermaßen den kleinsten gemeinsamen Nenner für sehr unterschiedliche stilistische Richtungen, deren Bandbreite Menzel anhand einiger Beispiele 
(Koyama Kiyoshige, Mamiya Michio, Ishiketa Mareo) plastisch vor Augen führt. Einige Besonderheiten des Hōgaku-Komponierens verdienen Erwähnung. So erfordert es eine intime Vertrautheit mit den Eigenarten der Instrumente, die zu erlangen für westlich ausgebildete Komponist*innen oft eine Herausforderung darstellt - Menzel schildert die Schwierigkeiten, denen die Koto-Spielerin Suzuki Kiyoko in den 1950er Jahren begegnete, als sie nicht in hōgaku beheimatete Komponisten zu gewinnen versuchte. Auf der anderen Seite entstanden zahlreiche Kompositionen aus der HōgakuSzene heraus, die kaum Bezug zu den Entwicklungen und Diskursen der neuen Musik in und außerhalb Japans haben.

Einen Kulminationspunkt der Begegnung zwischen westlicher Avantgarde und hōgaku bildet sicherlich Takemitsu Tōrus November Steps für biwa, shakuhachi und Orchester (1967), uraufgeführt im Rahmen der 125-Jahrfeier der New Yorker Philharmoniker. Gerade der Erfolg dieses Werkes, so Menzel, trug allerdings dazu bei, dass erneut eine Diskussion über die vermeintliche Unvereinbarkeit von 'westlicher` und ’östlicher`Musikästhetik aufflammte, eine Diskussion, die sich seinerzeit zwar als anschlussfähig an andere (höchst problematische) Diskurse innerhalb Japans (wie die 'Japaner-Diskurse $[\text { nihonjinron }]^{9}$ ) erwies, deren inhaltliche Relevanz Menzel aber zu Recht bestreitet.

In den Schlussbetrachtungen macht Menzel auf den, alles in allem, durchaus ambivalenten Charakter der Hōgaku-Entwicklung aufmerksam: Es lässt sich nicht übersehen, dass hōgaku heute trotz aller Metamorphosen zu einer Minderheitenmusik geworden ist, die keineswegs breit in der Gesellschaft verwurzelt ist. Für die Musikwissenschaft freilich ist das Studium der neueren Hōgaku-Geschichte außerordentlich interessant - irgendwo im sNiemandsland zwischen Ethnomusikologie und Historischer

9 Theorien, die vor allem während der 1970er und 1980er Jahre versuchten, eine kulturelle, gesellschaftliche und sogar neurologische Einzigartigkeit von Japaner*innen zu beweisen; sie finden mittlerweile auch innerhalb Japans keine wissenschaftliche Akzeptanz mehr.
Musikwissenschaft angesiedelt, zwingt diese Geschichte dazu, die Grenzen dieser beiden Disziplinen zu hinterfragen.

Einige kleine Anmerkungen zu Menzels insgesamt höchst lesenswertem Buch. Es bleiben sweiße Flecken ‘ auf der Landkarte - über die Entwicklungen in gagaku und nō im 20. Jahrhundert erfährt man sehr wenig, aber auch Gagaku-Musiker*innen komponierten zuweilen neue Werke oder wirkten an deren Entstehung mit. Daneben fanden parallel zur Begegnung der traditionellen japanischen mit der westlichen Musik bereits vor dem Zweiten Weltkrieg Ansätze zu einer Öffnung der ryūha statt, was allerdings erst in Ansätzen erforscht ist. ${ }^{10}$

Auf zwei möglicherweise zu Missverständnissen führende kleine Irrtümer sei hingewiesen. William E. Caplin erscheint als Edward E. Caplin $(95 ;$ 316). Ferner wird im Zusammenhang mit dem gakusō - dem koto des gagaku, dessen Spielweise sich von der des sōkyokukotos (zokusō) deutlich unterscheidet - der Name Mitsuzaki Kengyō genannt (120), es kann sich aber nur um Yoshizawa Kengyō handeln (Yoshizawa Kengyō evoziert in seinen Werken mit dem gakusō assoziierte Spielfiguren, obwohl seine Werke der sōkyoku zuzurechnen sind).

Ein wenig ist daneben zu bedauern, dass im Gegensatz zu den üblichen Gepflogenheiten klassische Hōgaku-Stücke nur nach Aufnahme, ohne Bezugnahme auf Tabulaturen transkribiert wurden, obwohl die Tabulaturen doch eine gewisse Vorstellung vom metrischen Empfinden der Hōgaku-Musiker*innen vermitteln sowie auditiv nicht immer zuverlässig zu erschließende Spieltechniken erkennen lassen (dies gilt insbesondere für die Transkription der Anfangstakte von Yaegoromo, 85).

Schließlich möchte sich der Rezensent als ausgesprochener Liebhaber klassischer (also vor der Meiji-Zeit entstandener) jiuta-sōkyoku nicht Menzels Hypothese anschließen, »dass hinter den Repertoireerweiterungen und -transformationen der sōkyoku-Tradition [...] in fast allen Fällen Marktstrategien standen« (64). Dazu scheinen mir gerade im klassischen jiutasōkyoku Personalstil und Experimentierlust der 
Komponisten zu ausgeprägt. So findet man z. B. bei Matsuura Kengyō (in Shiki-no-nagame) eine Passage, die man kaum anders als atonal nennen kann; Yoshizawa Kengyō erfand eine völlig neue Skala für seine Werke; Mitsuzaki Kengyō modifizierte ein grundlegendes Formelement des klassischen jiuta-sōkyoku, die kakeai (Frage-Antwort-Spiele), indem er sie in komplexer Asymmetrie gestaltete, und entwi- ckelte um die Mitte des 19. Jahrhunderts eine eigenständige japanische Polyphonie...

Doch sind diese Einwände letztlich unerheblich angesichts der grundlegenden Bedeutung von Menzels Arbeit für ein vertieftes Verständnis der japanischen Musiklandschaft im 20. Jahrhundert.

\section{Hubertus Dreyer}

\section{Literatur}

Fukuda, Chie (2015), »Sankyokur ni mirareru sankyoku-ensōkai no hensen. 1922 nen kara 44 nen no ensōkai-jōhō wo tōshite [Der Wandel der Sankyoku-Konzerte im Spiegel der Zeitschrift Sankyoku. Dargestellt anhand der Konzertankündigungen zwischen 1922 und 1944] «, Tōyō ongaku kenkyū 80, 21-38.

Fritsch, Ingrid (1979) Die Solo-Honkyoku der Tozan-Schule: Musik fur Shakuhachi zwischen Tradition und Moderne Japans, Kassel: Bärenreiter.

Harich-Schneider, Eta (1973), A History of Japanese Music, London: Oxford University Press.

Machida, Kashō (1956), Shamisen-seikyoku ni okeru senritsukei no kenkyū [Studien zu melodischen Patterns in shamisen-begleiteter Gesangsmusik], [Tokyo]: o. V.

Kikkawa, Eishi (1965), Nihon-ongaku no rekishi [Geschichte der japanischen Musik], Osaka: Sōgensha.

Kikkawa, Eishi (1984), Vom Charakter der japanischen Musik, übers. von Petra Rudolph, Kassel: Bärenreiter.
Provine, Robert C. / Yosihiko Tokumaru / J. Lawrence Witzleben (Hg.) (2002), The Garland Encyclopedia of World Music, Bd. 7: East Asia: China, Japan, and Korea, New York: Roudledge.

Yamada, Chieko / Mariko Ōkubo (Hg.) (2015), Shamisenongaku no senritsukei-kenkyū Machida Kashō wo megutte [Studien zu melodischen Patterns in Shamisen-Musik anknüpfend an Machida Kashō], Kyōto Shiritsu Geijutsu Daigaku: Nihon dentō ongaku kenkyū sentā.

Yakō, Masato (2002), Shamisen-ongaku he no atarashii apurōchi. Keisanki wo mochiita bunseki no kanōsei [Ein neuer Zugang zur Shamisen-Musik. Über Möglichkeiten computergestützter Analyse], in: Nihon no katarimono. Kōtōsei, kōzō, igi [Die narrative Musik Japans. Mündliche Überlieferung, Struktur, Bedeutung], hg. vom Nichibunken sōsho, Kyōto: Kokusai Nihon bunka sentā, 249-265. http://publications.nichibun.ac.jp/region/d/NS H/series/niso/2002-10-31/s001/s037 /pdf/article.pdf (12.10.2018)

Dreyer, Hubertus (2018): Stefan Menzel, Hōgaku. Traditionelle japanische Musik im 20. Jahrhundert, Hildesheim: Olms 2015. ZGMTH 15/2, 225-229.

https://doi.org/10.31751/983

(C) 2018 Hubertus Dreyer (hubertus@temporubato.com)

Dieser Text erscheint im Open Access und ist lizenziert unter einer

Creative Commons Namensnennung 4.0 International Lizenz.

This is an open access article licensed under a

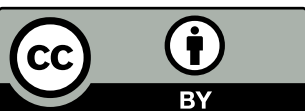

Creative Commons Attribution 4.0 International License.

eingereicht / submitted: 30/09/2018

angenommen / accepted: 01/10/2018 veröffentlicht / first published: 18/12/2018

zuletzt geändert / last updated: 18/12/2018 